\section{Autopercepção e condições de saúde bucal em uma população de idosos}

\author{
Self-perception and oral health conditions \\ in an elderly population
}

\author{
${ }^{1}$ Faculdade de Odontologia \\ de Piracicaba, Universidade \\ Estadual de Campinas, \\ Piracicaba, Brasil. \\ Correspondência \\ M. L. R. Sousa \\ Departamento de \\ Odontologia Social, \\ Faculdade de Odontologia \\ de Piracicaba, Universidade \\ Estadual de Campinas. \\ Av. Limeira 901, \\ Piracicaba, $S P$ \\ 13414-018, Brasil. \\ luzsousa@fop.unicamp.br
}

\begin{abstract}
This study assessed oral health conditions through clinical examination and self-perception. The sample consisted of 112 subjects 60 years of age or older in Rio Claro, São Paulo, Brazil, divided into two groups: $G 1$, with dental insurance ( $n=$ $55)$ and G2, without dental insurance $(n=57)$. Clinical examinations were conducted according to WHO guidelines. Data on self-perceived oral health were collected using the GOHAI (Geriatric Oral Health Assessment Index). DMFT was 29.13, higher in G1 ( $p=0.0091)$. Mean number of teeth currently present in both groups was 7.63 , higher in $G 2(p=0.0131)$. Periodontal conditions showed a high percentage of null sextants (70.3\%), where CPI revealed higher prevalence for calculus and PIP for pockets up to $3 \mathrm{~mm}$. The percentage of edentulous subjects was 45.5\%, higher in $G 1$ ( $p=0.0142)$. Among edentulous subjects, $69.6 \%$ wore complete upper dentures and $42.9 \%$ complete lower dentures. Mean GOHAI was 33.61, qualifying self-perceived oral health status as positive, and the only difference was in the physical dimension, where G2 showed higher values ( $p=0.0154)$. Self-perceived oral health was considered satisfactory, but this was not confirmed by clinical data from the two groups.
\end{abstract}

Oral Health; Self-Concept; Aged
Débora Dias da Silva 1

Maria da Luz Rosário de Sousa 1

Ronaldo Seichi Wada 1

\section{Introdução}

Nas últimas décadas, o envelhecimento populacional no Brasil vem ocorrendo de forma crescente, sendo um dos fenômenos demográficos mais importantes da atualidade.

Entretanto, o quadro epidemiológico e a ausência de programas voltados para a população idosa brasileira caracterizam a condição de saúde bucal precária encontrada nesse grupo 1 .

A obtenção de dados epidemiológicos é importante, pois esses quantificam as condições de saúde bucal dos indivíduos, além de serem usados no planejamento, organização e monitoramento dos serviços de saúde prestados 2 . Entretanto, baseiam-se em índices que fornecem dados quantitativos, levando em conta apenas a visão do profissional. Atualmente, além da obtenção dos dados quantitativos, a tendência é que também sejam obtidos dados qualitativos, através da autopercepção, na qual o próprio indivíduo percebe suas condições de saúde bucal e as necessidades de tratamento 3,4 .

A maioria dos estudos que avaliam mudanças no estado de saúde bucal de indivíduos e populações tem sido baseada em indicadores clínicos da doença, existindo relativamente poucas avaliações relativas à sua saúde e bem-estar a partir da percepção do indivíduo 5 .

Apesar do exposto anteriormente, é crescente o interesse em estudar a influência da condição dos dentes na qualidade de vida dos 
indivíduos, não somente relacionados com as conseqüências físicas, mas incluindo também as sociais e psicológicas (psicossociais) 6. Estas avaliações, realizadas através da autopercepção, fornecem informações diferentes das obtidas pela avaliação clínica, realizada pelo profissional 7,8 .

Foram então desenvolvidos vários instrumentos para obtenção de dados sobre autopercepção, dentre eles, o Geriatric Oral Health Assessment Index (GOHAI), que foi utilizado neste estudo 9 . Apesar de originalmente ter sido desenvolvido para avaliar o impacto das doenças bucais em populações idosas, também tem sua indicação para adultos de todas as idades 10 .

Em acréscimo, os estudos epidemiológicos em saúde bucal também têm sido complementados freqüentemente com dados sobre condições sócio-econômicas, levando em consideração a importância da influência dessas nas doenças e condições de saúde, sendo consideradas como fatores determinantes de saúde e de acesso a serviços 11,12.

Por se entender que o Município de Rio Claro, São Paulo, Brasil, apresenta uma população com uma porcentagem significativa de indivíduos idosos, ou seja, 11,0\% são indivíduos com idade acima de 60 anos 13, torna-se importante conhecer a condição de saúde bucal desses.

Assim, os objetivos deste estudo foram verificar a saúde bucal e a autopercepção em relação à saúde bucal em indivíduos idosos, com e sem acesso a tratamento odontológico conveniado, e dentre estes, aqueles com e sem dentes naturais, avaliando também as variáveis sócio-demográficas dessa população.

\section{Métodos}

Este estudo, caracterizado como sendo do tipo transversal, teve seu início após ser aprovado pelo Comitê de Ética em Pesquisa da Faculdade de Odontologia de Piracicaba (FOP) da Universidade Estadual de Campinas (UNICAMP).

O tamanho da amostra foi definido considerando-se a estimativa da doença cárie, levando-se em conta a média e o desvio padrão da experiência de cárie (índice CPO-D), na qual a amostra adequada para representar a população de referência, considerando-se a experiência de cárie, deveria ser de, no mínimo, 14 indivíduos para a região Sudeste, segundo critérios adotados para o levantamento das Condições de Saúde Bucal da População Brasileira - Projeto SB Brasil 2003 11. Neste estudo, como haveria estratificações em grupos, a amostra foi aumentada.
Para a seleção da amostra, houve, a princípio, uma pré-seleção dos indivíduos quanto à idade (mais de 60 anos) e ter acesso ou não a tratamento odontológico conveniado. Essa variável foi caracterizada pelos autores através de uma lista de indivíduos idosos que, certamente, faziam parte de um convênio odontológico oficializado. Foram obtidas, então, duas listas, uma fornecida pelo próprio convênio, que constou de 203 indivíduos com idade acima de 60 anos, e outra, pela prefeitura local com 34 grupos de terceira idade, para a seleção dos que não possuíam esse tipo de convênio.

Em seguida, foi feito contato telefônico com os indivíduos conveniados sorteados ( $\mathrm{n}=77$ ), dos quais, 57 concordaram em participar. Quanto àqueles que freqüentavam grupos de terceira idade, o contato foi realizado pessoalmente durante a visita do próprio pesquisador aos locais sorteados $(n=12)$, sendo selecionados 189 indivíduos, dos quais, 55 concordaram em participar.

Assim, obteve-se a amostra final de 112 indivíduos com idade acima de 60 anos, de ambos os sexos, moradores da cidade de Rio Claro, sendo dividida em dois grupos: G1 - indivíduos que relataram não ter acesso a tratamento odontológico conveniado ( $n=55)$ e G2 - indivíduos que relataram ter acesso a tratamento odontológico conveniado $(\mathrm{n}=57)$. Além dessa estratificação, foi realizada uma segunda, quanto à presença ou ausência de dentes naturais, para verificar a distribuição das condições estudadas e diferenças entre estes grupos (com e sem acesso a tratamento odontológico; com e sem dentes), referentes às condições de saúde bucal, sócio-demográficas e autopercepção.

O processo de calibração foi realizado num período de vinte horas, sendo que a própria pesquisadora foi capacitada para a coleta de dados. Durante esse processo, foi medido o erro intra-examinador antes da coleta de dados com o índice de concordância kappa (exame clínico), com valores de 0,95 para cárie, 0,53 para CPI (Índice Periodontal Comunitário) e PIP (Perda de Inserção Periodontal) e 1,0 para uso e necessidade de próteses; a porcentagem de concordância foi de 73,0\% (CPI e PIP), 96,0\% (cárie) e 100,0\% (uso e necessidade de próteses), sendo consideradas moderada, excelente e perfeita concordância, respectivamente 14 .

Após a obtenção do termo de consentimento livre e esclarecido, assinado pelo voluntário, concordando em participar deste estudo, os dados foram coletados entre os meses de abril e agosto de 2003.

O exame epidemiológico foi realizado sob iluminação natural, com o uso de espelhos bu- 
cais, espátulas de madeira e sondas periodontais CPI, como preconizado pela Organização Mundial da Saúde (OMS) 2. O examinador encontrava-se posicionado em frente ao voluntário, e o anotador, ao lado, ambos sentados em cadeiras.

Para avaliar as condições dos dentes e necessidade de tratamento, foram registrados códigos e critérios preconizados pela OMS 2, adaptados pela Faculdade de Saúde Pública (FSP) da Universidade de São Paulo (USP) 15, através do índice CPO-D, que totaliza o número de dentes cariados, perdidos e obturados.

O CPI foi avaliado usando-se três indicadores das condições periodontais: sangramento gengival, cálculo e bolsas periodontais. Realizou-se também a avaliação da PIP, obtendo, assim, uma estimativa da destruição acumulada durante a vida útil da inserção periodontal. Tanto para o CPI como para o PIP, foram utilizadas sondas milimetradas preconizadas pela OMS.

Com relação ao uso e à necessidade protética, foi realizado um registro tanto para a maxila quanto para a mandíbula, com relação ao tipo de prótese. Entretanto, neste estudo, os relatos foram com relação ao uso e necessidade de próteses totais, pelo fato dessa condição representar as maiores prevalências quanto ao uso.

Quando da coleta de dados epidemiológicos, houve $10,0 \%$ de reexames (somente em indivíduos dentados) para o cálculo do índice de concordância kappa e da porcentagem de discordância (exame clínico), verificando, assim, a confiabilidade dos resultados.

Foram considerados critério de exclusão do voluntário a incapacidade para o exame clínico e também a idade (possuir menos de 60 anos).

As questões do índice GOHAI, feitas por meio de entrevista, envolveram a análise de informações proporcionadas pelos próprios indivíduos quanto à influência de seus problemas de saúde bucal nas dimensões: física/funcional - incluindo alimentação, fala e deglutição; psicossocial/psicológica - compreendendo preocupação ou cuidado com a própria saúde bucal, insatisfação com a aparência, autoconsciência relativa à saúde bucal e o fato de evitar contatos sociais devido a problemas odontológicos e relativos à dor/desconforto - considerando o uso de medicamentos para aliviar essas sensações, desde que provenientes da boca 7 .

Para as respostas destas questões (sempre, às vezes e nunca), existem pesos/escores (1, 2 e 3 , respectivamente), que somados darão o valor do índice, que quanto maior, melhor a saúde bucal do indivíduo e, conseqüentemente, melhor a qualidade de vida $1,3,7$, sendo que os valores variaram de 12 a 36 , classificados como alto (34 a 36), moderado (31 a 33) e baixo (menor que 30).

As questões que avaliaram condições sócio-demográficas foram relativas à renda familiar (total de rendimentos em salário mínimo), ao grau de escolaridade (anos de estudo), ao número de filhos e ao gênero (homens e mulheres), para a comparação entre os grupos.

\section{Análise estatística}

A análise dos dados foi realizada de forma descritiva, apresentando-se os resultados do GOHAI e suas dimensões em cada grupo, ressaltando qual dimensão foi mais relevante. Foram utilizados os testes Mann Whitney e de proporção para a comparação entre variáveis clínica e de autopercepção, além das variáveis sócio-demográficas entre os grupos e no grupo como um todo. Para comparar a média de idade entre os grupos, utilizou-se o teste $t$.

\section{Resultados}

O índice de concordância kappa, realizado durante a coleta de dados, foi de 0,97 para cárie, 0,86 para PIP, 0,88 para o CPI, e para uso e necessidade de próteses, o valor foi de 1,0, comprovando que houve uma porcentagem alta de concordância, portanto, os resultados são confiáveis 14 .

Houve uma perda de aproximadamente $26,0 \%$ na amostra no G2 e no G1, essa perda aumentou atingindo mais de $70,0 \%$.

Foram examinados 112 indivíduos, sendo $49,1 \%(n=55)$ no G1 (sem acesso a tratamento odontológico conveniado) e 50,9\% ( $\mathrm{n}=57$ ) no G2 (com acesso a tratamento odontológico conveniado). Nos grupos G1 e G2, a maior porcentagem foi relativa ao gênero feminino, sendo estatisticamente maior no G1 ( $p=0,0000)$; a idade média foi de 69,33 e 69,75 anos no G1 e G2, respectivamente, não apresentando diferença estatisticamente significante $(p=0,7055)$ (Tabela 1).

A estratificação quanto à presença ou não de dentes, no grupo, foi de $45,5 \%$ ( $n=61$ ) dentados e $54,5 \%(n=51)$ edêntulos, sendo que estes apresentaram maior proporção de mulheres que os dentados ( $p=0,0214)$, não apresentando diferença estatística quando se comparou as idades ( $\mathrm{p}=0,2238)$ ( Tabela 2$)$.

A escolaridade, baseada em anos de estudo, apresentou média maior no G2 e nos indivíduos dentados $(\mathrm{p}<0,05)$. Com relação ao número de filhos, esse foi estaticamente maior no 
Tabela 1

Variáveis clínicas, sócio-demográficas, índice GOHAl e suas dimensões em indivíduos com mais de 60 anos, segundo grupos com e sem acesso a tratamento odontológico conveniado. Rio Claro, São Paulo, Brasil, 2003.

\begin{tabular}{|c|c|c|c|c|c|c|c|}
\hline \multirow[t]{3}{*}{ Variáveis } & \multicolumn{7}{|c|}{ Grupos } \\
\hline & \multicolumn{2}{|c|}{$\mathrm{G} 1(\mathrm{n}=55)$} & \multicolumn{2}{|c|}{$\mathrm{G} 2(\mathrm{n}=57)$} & \multicolumn{2}{|c|}{$\mathrm{GG}(\mathrm{n}=112)$} & \multirow{2}{*}{$\mathrm{p}$} \\
\hline & $\mathrm{n}$ & $\%(D P)$ & $\mathrm{n}$ & $\%(D P)$ & $\mathrm{n}$ & $\%(\mathrm{DP})$ & \\
\hline CPO-D (média) & 29,93 & $(3,49) a$ & 28,37 & $(3,85) \mathrm{b}$ & 29,13 & $(3,75)$ & $0,0091^{\star}$ \\
\hline Edentulismo & 32 & $58,2 \mathrm{a}$ & 19 & $33,3 b$ & 51 & 45,5 & $0,0142^{\star \star}$ \\
\hline Uso de prótese total superior & 43 & 78,2 & 35 & 61,4 & 78 & 69,6 & $0,0845^{\star *}$ \\
\hline Uso de prótese total inferior & 30 & $54,5^{\mathrm{a}}$ & 18 & $31,6 b$ & 48 & 42,9 & $0,0235^{\star \star}$ \\
\hline Necessidade de prótese total superior & 2 & 3,6 & 1 & 1,8 & 3 & 2,7 & $0,9749 * \star$ \\
\hline Necessidade de prótese total inferior & 5 & 9,1 & 1 & 1,8 & 6 & 5,4 & $0,1922^{\star \star}$ \\
\hline Necessidade de tratamento & 13 & 23,6 & 13 & 22,8 & 26 & 23,2 & $0,9045^{\star \star}$ \\
\hline Dentes presentes (média) & 5,30 & $(7,72)^{a}$ & 9,86 & $(9,87)^{\mathrm{b}}$ & 7,63 & $(9,13)$ & 0,0131 * \\
\hline Dentes hígidos (média) & 1,67 & $(3,01) a$ & 3,10 & $(3,63) b$ & 2,40 & $(3,40)$ & $0,0170^{\star}$ \\
\hline GOHAl (média) & 33,25 & $(2,91)$ & 33,95 & $(3,11)$ & 33,61 & $(3,02)$ & $0,1133^{*}$ \\
\hline Dimensão física (média) & 10,78 & $(1,29) a$ & 11,30 & $(1,13)^{b}$ & 11,04 & $(1,23)$ & $0,0154^{*}$ \\
\hline Dimensão psicossocial (média) & 14,04 & $(1,45)$ & 14,25 & $(1,60)$ & 14,14 & $(1,52)$ & $0,4594^{*}$ \\
\hline Dor/desconforto (média) & 8,45 & $(0,94)$ & 8,44 & $(0,93)$ & 8,45 & $(0,93)$ & $0,6142^{\star}$ \\
\hline Idade (média) & 69,33 & $(5,86)$ & 69,75 & $(6,06)$ & 69,54 & $(5,94)$ & $0,7055^{\star \star \star}$ \\
\hline Mulheres & 50 & $90,9 a$ & 29 & $50,9 b$ & 79 & 70,5 & $0,0000^{* *}$ \\
\hline Escolaridade (média) & 3,44 & $(3,63) a$ & 4,88 & $(2,90) \mathrm{b}$ & 4,18 & $(3,34)$ & $<0,0001^{\star}$ \\
\hline Número de filhos (média) & 4,85 & $(3,65) \mathrm{a}$ & 3,09 & $(1,96)^{b}$ & 3,96 & $(3,04)$ & $0,0099 *$ \\
\hline Renda familiar (média) & 4,04 & $(5,46)^{a}$ & 3,54 & $(1,68)^{b}$ & 3,78 & $(3,98)$ & $0,0044^{*}$ \\
\hline
\end{tabular}

** Teste de proporção;

* Mann Whitney

*** Teste $t$, com $5 \%$ de significância;

Nota: Números seguidos de letras diferentes na horizontal diferem estatisticamente entre si, considerando cada variável.

Tabela 2

Variáveis clínicas, sócio-demográficas, índice GOHAl e suas dimensões em indivíduos com mais de 60 anos, segundo grupos de dentados e desdentados. Rio Claro, São Paulo, Brasil, 2003.

\begin{tabular}{|c|c|c|c|c|c|c|c|}
\hline \multirow[t]{3}{*}{ Variáveis } & \multicolumn{7}{|c|}{ Grupos } \\
\hline & \multicolumn{2}{|c|}{ Dentados $(n=61)$} & \multicolumn{2}{|c|}{ Desdentados $(n=51)$} & \multicolumn{2}{|c|}{$\mathrm{GG}(n=112)$} & \multirow[t]{2}{*}{ Valor de $\mathrm{p}$} \\
\hline & $\mathrm{n}$ & $\%(d p)$ & $\mathrm{n}$ & $\%(d p)$ & $\mathrm{n}$ & $\%(d p)$ & \\
\hline Necessidade de prótese total superior & 2 & 3,3 & 1 & 2,0 & 3 & 2,7 & $0,8749 *$ \\
\hline Necessidade de prótese total inferior & 1 & 1,6 & 5 & 9,8 & 6 & 5,4 & $0,1363^{*}$ \\
\hline Necessidade de tratamento & 26 & 42,6 & - & - & 26 & 23,2 & $0,7665^{\star}$ \\
\hline Mulheres & 37 & $60,7 a$ & 42 & $82,4 b$ & 79 & 70,5 & $0,0214^{*}$ \\
\hline Idade (média) & 68,92 & $(5,68)$ & 70,29 & $(6,21)$ & 69,54 & $(5,94)$ & $0,2238^{* *}$ \\
\hline Escolaridade (média) & 4,97 & $(3,57) a$ & 3,25 & $(2,80)^{b}$ & 4,18 & $(3,34)$ & $0,0026^{* \star *}$ \\
\hline Número de filhos (média) & 3,54 & $(2,60)$ & 4,48 & $(3,47)$ & 3,96 & $(3,04)$ & $0,1464^{* * *}$ \\
\hline Renda familiar (média) & 4,74 & $(4,93)^{a}$ & 2,62 & $(1,85)^{b}$ & 3,78 & $(3,98)$ & $<0,0001^{\star * *}$ \\
\hline GOHAI (média) & 34,07 & $(2,71)$ & 33,06 & $(3,29)$ & 33,61 & $(3,02)$ & $0,1126^{* * *}$ \\
\hline Dimensão física (média) & 11,33 & $(1,06)^{a}$ & 10,71 & $(1,35)^{b}$ & 11,04 & $(1,23)$ & $0,0089 * * *$ \\
\hline Dimensão psicossocial (média) & 14,31 & $(1,36)$ & 13,94 & $(1,69)$ & 14,14 & $(1,52)$ & $0,1872^{\star \star \star}$ \\
\hline Dor/desconforto (média) & 8,49 & $(0,91)$ & 8,39 & $(0,96)$ & 8,45 & $(0,93)$ & $0,8309 * * *$ \\
\hline
\end{tabular}

* teste de proporção;

** teste t, com $5 \%$ de significância:

$\star \star \star$ Mann Whitney;

Nota: Números seguidos de letras diferentes na horizontal diferem estatisticamente entre si, considerando cada variável. 
G1 em relação ao G2 ( $p=0,0099)$, não sendo diferente nos grupos de dentados e edêntulos ( $\mathrm{p}=$ 0,1464) (Tabelas 1 e 2).

Com relação à renda, calculada em termos de salário mínimo, a média foi maior no G1 e nos indivíduos dentados $(\mathrm{p}<0,05)$ (Tabelas 1 e 2).

A experiência de cárie foi de 29,93 no G1 e 28,37 no G2, sendo significantemente maior no $\mathrm{G} 1$ ( $\mathrm{p}=0,0091)$; a porcentagem de edentulismo foi de $58,2 \%(n=32)$ e $33,3 \%(n=19)$ nos grupos G1 e G2, respectivamente, sendo que, no G1, foi significantemente maior que no G2 ( $p=0,0142)$ (Tabela 1).

A média de dentes presentes foi de 5,31 no G1 e 9,86 no G2, sendo estatisticamente maior no G2 ( $\mathrm{p}=0,0131)$. O G1 apresentou, em média, 1,67 dentes hígidos; e no G2, essa média foi de 3,11 , sendo estatisticamente maior que no G1 ( $\mathrm{p}=0,0170)$ (Tabela 1$)$.

O número de sextantes excluídos, ou seja, que não possuíam dentes para o exame do CPI e PIP, foi elevado no grupo geral, sendo de $70,2 \%$, com uma média de 3,51 por indivíduo.

Para o CPI, depois dos sextantes excluídos, as maiores condições foram sextantes com dentes hígidos $(16,0 \%)$, seguidos dos dentes com cálculo $(9,0 \%)$, sangramento e bolsas com mais de $4 \mathrm{~mm}$ somam 4,7\%. Quanto aos grupos (possuir ou não acesso a tratamento odontológico conveniado), essas condições foram de 79,6\% e 61,0\% de sextantes excluídos, 10,3\% e 21,7\% hígidos; $6,2 \%$ e $11,9 \%$ com tártaro e $3,9 \%$ e $5,4 \%$ para as demais condições, nos G1 e G2, respectivamente.

As condições mais prevalentes para o PIP, complementando os sextantes excluídos (70,3\%), foram os sextantes com perdas de inserção entre 0 e $3 \mathrm{~mm}(21,2 \%)$, perdas entre 4 e $5 \mathrm{~mm}(5,1 \%)$ e as perdas maiores que $6 \mathrm{~mm}(3,4 \%)$. Nos grupos G1 e G2, essas condições foram, respectivamente, de 79,6\% e 61,0\% sextantes excluídos, $14,0 \%$ e $28,0 \%$ de perdas de inserção entre 0 e $3 \mathrm{~mm}, 5,0 \%$ para perdas entre 4 e $5 \mathrm{~mm}$ (G1 e G2), $1,3 \%$ e $5,3 \%$ de perdas entre 6 e $8 \mathrm{~mm}$, e as demais condições foram menos que $1,0 \%$.

Houve uma grande porcentagem de indivíduos que usavam próteses totais superior e inferior e, quando foram comparadas essas porcentagens nos grupos, houve diferença estatisticamente significante somente em relação ao uso de prótese total inferior ( $\mathrm{p}=0,0235)$, sendo maior no G1 (Tabela 1).

Quanto à necessidade de próteses, tanto para a arcada superior quanto para a inferior, apenas $2,7 \%$ e $5,4 \%$ do total de indivíduos apresentavam essas necessidades, ou seja, a maioria não necessitava de próteses totais, não havendo diferenças entre os grupos ( $p>0,05)$ (Tabela 1).
Os valores médios do GOHAI foram 33,25 e 33,95 nos G1 e G2, respectivamente, não havendo diferença estatisticamente significante $(\mathrm{p}=$ 0,1133). Quando avaliado em suas dimensões, não houve diferença significante entre os grupos com relação à dor/desconforto $(p=0,6142)$ e psicossocial ( $\mathrm{p}=0,4594$ ), porém, o G1 apresentou média significantemente menor $(\mathrm{p}=$ 0,0154) que o G2 com relação à dimensão física (Tabela 1).

As questões relacionadas com a dimensão física foram avaliadas uma a uma para verificar em qual(is) delas haveria diferença entre os grupos G1 e G2, sendo que a questão “Teve problemas mordendo ou mastigando alimentos sólidos como carne ou maçã?" apresentou-se muito próxima do nível de significância de $5 \%$ (p = 0,0530).

Porém, quando da estratificação do grupo com relação à presença ou não de dentes, houve diferença estatisticamente significante na dimensão física, na qual os dentados obtiveram uma média maior $(\mathrm{p}=0,0089)$ comparada com a dos desdentados, sendo que a questão com diferença estatística entre esses grupos também foi: "Teve problemas mordendo ou mastigando alimentos sólidos como carne ou maçã?", na qual os desdentados apresentaram menos respostas positivas que os dentados $(\mathrm{p}=$ 0,0470) (Tabela 2).

Quanto à necessidade de tratamento, não houve diferença entre os grupos G1 e G2 (p > $0,05)$ (Tabela 1).

\section{Discussão}

As limitações deste estudo deveram-se ao fato de que, no grupo G1, houve quase que exclusivamente mulheres e a maior perda amostral (70,0\%), entretanto, no grupo como um todo, os resultados podem ser extrapolados para a população idosa de Rio Claro, pois se sabe que a expectativa de vida para as mulheres é maior 16,17 , e muitos trabalhos relatam dados com maior porcentagem de mulheres 18,19,20,21,22. Torna-se necessário enfatizar que, no presente estudo, o fato do G1 representar grupos de terceira idade já indica uma maior tendência da participação de mulheres.

O fato de os autores terem uma lista dos indivíduos idosos conveniados não impossibilita que alguns indivíduos do G1, ou seja, pertencentes a grupos de terceira idade (considerados sem acesso a tratamento odontológico conveniado), pudessem ter esse acesso a tratamento odontológico (conveniado ou não), e pôde ser verificado em torno de $20,0 \%(n=12)$ dos rela- 
tos. Isso pode também ter interferido nos resultados, no entanto, como esta resposta era subjetiva, não haveria possibilidade de confirmação, e os autores assumiram a divisão da amostra dessa forma. Assim, o G1 pode representar a saúde bucal dos idosos.

Em acréscimo, não foi realizado cálculo do tamanho amostral ideal para a coleta de dados sobre autopercepção e houve uma alta porcentagem de perda amostral pela recusa dos indivíduos a participarem deste estudo, que provavelmente ocorreu pela própria condição bucal apresentada (a maior parte de edêntulos, e muitos deles, apesar de usarem próteses, sentiamse constrangidos por esse fato).

Os resultados das variáveis clínicas, neste estudo, não diferem dos encontrados por Padilha \& Souza 23, Castellanos-Fernandes et al. 24 , Silva 25, Carneiro 20 e Cangussu et al. 26, nos quais o CPO-D e a porcentagem de edentulismo foram altos, apresentando poucos dentes presentes. Como no presente estudo, em todos os trabalhos citados, a maior porcentagem era de mulheres, e a idade ou média de idade era superior a 60 anos.

Ainda quando comparados com dados do mesmo município (Rio Claro) que datam de 1998 27, percebe-se que não houve mudanças no quadro epidemiológico, o que reforça a atenção inadequada para esse grupo.

Porém, quando comparados com estudos internacionais, estes valores são mais elevados que alguns 28,29, mas semelhantes a outros 30,31. O que indica que não somente as condições sócio-econômicas, mas também os padrões culturais e dietéticos, como na China 29 e Finlândia 31 , além da realização desses estudos em épocas distintas, como na Austrália 28 e nos Estados Unidos 30, podem influenciar nas condições de saúde bucal.

A porcentagem de sextantes nulos e dos que usavam próteses foram altas devido ao número de edêntulos, o que corrobora com dados relatados por Rosa et al. 32 , Castellanos-Fernandes et al. 24 , Carneiro 20 e pela Secretaria de Estado da Saúde de São Paulo 33, o que implica admitir que houve falhas na atenção para esse grupo 34 , podendo ser evidenciada com o predomínio do tratamento mutilador.

Neste trabalho, o G1 (sem acesso a tratamento odontológico conveniado) apresentou piores condições em relação às variáveis de saúde bucal, comparando com o G2 (com acesso a tratamento odontológico conveniado), ou seja, CPO-D mais elevado, menor número de dentes presentes e hígidos, conseqüentemente, uma elevada porcentagem de edentulismo. Houve predominância de mulheres, além de o número de filhos ter sido maior, menor escolaridade e apesar da renda familiar ter sido maior para o $\mathrm{G} 1$, a realidade foi de uma renda entre 3,5 e 4 salários mínimos, ou seja, não caracterizando renda elevada em ambos os grupos.

Esses dados reforçam a importância de estudos que avaliam, além das variáveis sóciodemográficas, as influências culturais e dentais nas condições de saúde, e, conseqüentemente, na qualidade de vida de uma população e dentre grupos de uma mesma população 35 , pois, como relatado por Silva \& Castellanos-Fernandes 1 , o meio social tem influência no modo como o indivíduo pensa, sente e age com relação à sua saúde.

Quando foram comparados os dados deste estudo com os do Estado de São Paulo 33, que apresentou uma amostra representativa da população de referência, pois foi do tipo domiciliar, a situação foi muito próxima, com CPO-D alto $(28,18)$, baixa média de dentes presentes $(4,93)$ e hígidos $(2,96)$, alta porcentagem de dentes perdidos $(93,0 \%)$ e de indivíduos que usavam próteses totais superior (59,8\%) e inferior $(71,1 \%)$.

Não foram verificadas diferenças significativas nas dimensões psicossociais e com relação à dor/desconforto do índice GOHAI, no entanto, essa diferença existiu na dimensão física, podendo sugerir que somente os problemas relacionados à mastigação realmente os incomodaram nos últimos três meses. Esse fato pode ser atribuído a outras situações em que haja preocupação, que não sejam relacionadas à saúde bucal. Pode-se pensar na hipótese da necessidade de indicadores subjetivos (autopercepção) mais abrangentes para verificar bem-estar e qualidade de vida 8 , não só em relação à saúde bucal 36, para avaliar o que estes indivíduos sentem e percebem, como também absenteísmo ao trabalho (casos específicos para os idosos) ou a atividades cotidianas relacionadas à saúde bucal.

O grupo, como um todo, apresentou um valor considerado de moderado a alto com relação ao GOHAI (acima de 30), o que corresponde a uma percepção positiva das condições de saúde bucal (Figura 1), entretanto a realidade das condições clínicas mostrou-se um pouco diferente da autopercepção, o que pode ser confirmado com dados de Silva \& CastellanosFernandes 1; Jokovic \& Locker 37 e Matthias et al. 4, ou seja, ainda há um número elevado de indivíduos edêntulos que usam próteses, mas que se sentem apenas incomodados com os problemas de mastigação.

Alguns estudos descritos na literatura 1,38,39 avaliaram o índice GOHAI em grupos de indi- 
Porcentagem de indivíduos com mais de 60 anos que obtiveram valores acima e abaixo de 30,

relacionados ao índice GOHAl. Rio Claro, São Paulo, Brasil, 2003.

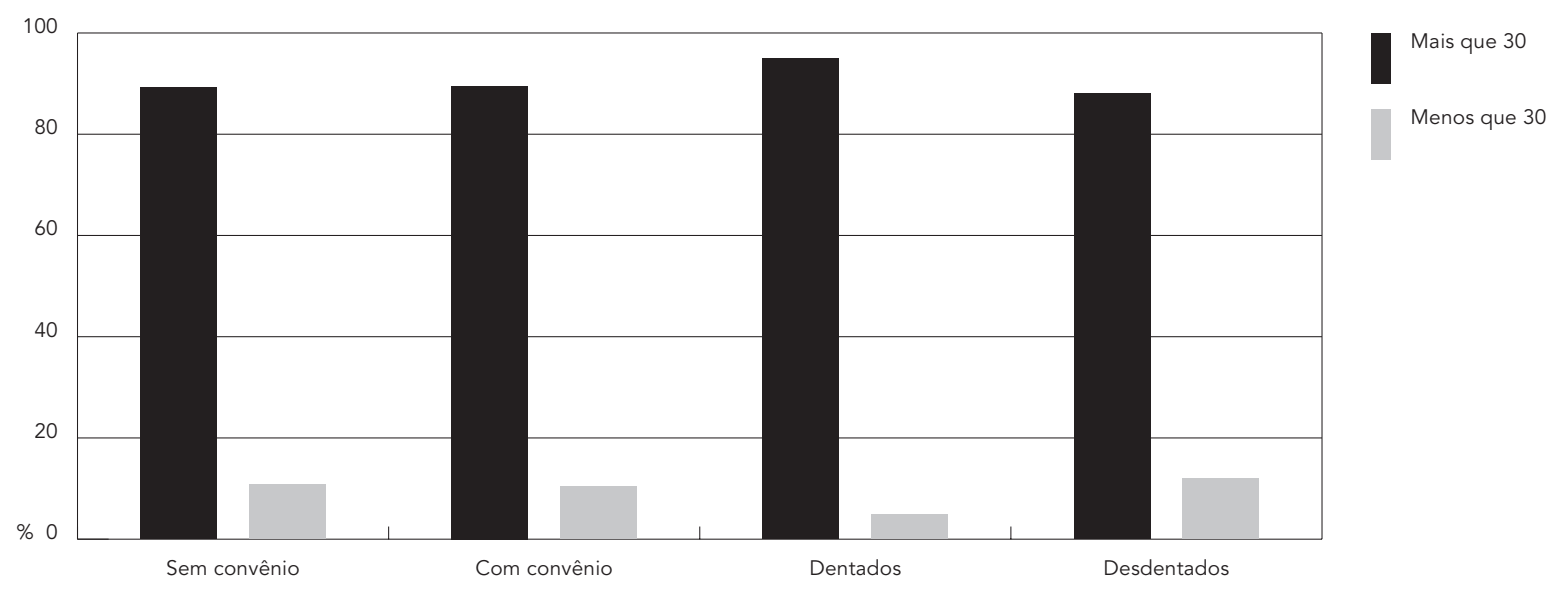

víduos dentados, o que infere dizer que os resultados do presente estudo foram influenciados pelo fato de haver também a inclusão de indivíduos edêntulos.

Por outro lado, há o questionamento que se realmente o uso de prótese total estaria interferindo nesses resultados (variável não avaliada neste estudo), pois, apesar de ter mais indivíduos que usam próteses inferiores no G1 e não haver diferenças quanto ao uso das superiores nos grupos, o G1 apresentou menos dentes presentes, menos sextantes hígidos, maior impacto negativo na dimensão física no GOHAI, apontando, dessa forma, para outros fatores que também podem influenciar nos resultados, tendo em vista que, mesmo com as respostas positivas para o índice GOHAI, sabe-se que o número de dentes tem relação direta com a qualidade de vida.

O valor médio do índice GOHAI, neste estudo, coincide com os relatados por Silva \& Castellanos-Fernandes 1 e Kressin et al. 7 , estando um pouco acima dos valores encontrados por Silva 40 e Mascarenhas 3 , tendo que ser vistos com cautela, pois as amostras são distintas do presente estudo.

Não houve diferença entre ser dentado ou edêntulo no grupo em relação ao índice GOHAI, somente na dimensão física. O fato de a maioria dos edêntulos estarem reabilitados, podendo ser evidenciado pela baixa porcentagem dos que necessitavam de próteses totais, fez com que estes não apresentassem problemas em relação às outras dimensões e, assim, propõe-se que mais trabalhos que verifiquem autopercepção em indivíduos reabilitados com próteses totais, confrontando com os dentados, usando outros indicadores com maior sensibilidade e especificidade como, por exemplo, o OHIP-14 (Oral Health Impact Profile) 36 sejam realizados.

Com os resultados deste estudo, reforça-se a importância que deve ser dada aos fatores que podem influenciar as condições de saúde bucal, entre eles: escolaridade, renda, gênero, que têm sido estudados; porém, estratificando-se em grupos, há possibilidade de refinar os achados sobre a saúde bucal desse grupo, que, mesmo sendo insatisfatória, pode ser relacionada com acesso a tratamento odontológico conveniado e ter ou não dentes naturais, conhecendo, assim, a realidade para que, por meio de planejamento adequado, esse grupo possa ser assistido de forma integral, com programas e tratamentos específicos.

\section{Conclusões}

Apesar de as condições de saúde bucal para esse grupo etário ainda apresentarem-se insatisfatórias, evidenciadas pelos dados clínicos, com CPO-D alto e grande número de indivíduos edêntulos, a autopercepção foi altamente po- 
sitiva, sendo que a dimensão física do índice GOHAI foi avaliada e considerada melhor naqueles indivíduos com melhores condições periodontais e que apresentaram dentes naturais.

O fato de o indivíduo ter acesso ao tratamento odontológico conveniado parece contribuir para melhores condições de saúde bucal, salientando que essa variável não foi avaliada de forma isolada.

\section{Resumo}

Este estudo avaliou as condições de saúde bucal clinicamente e através da autopercepção. A amostra foi de 112 indivíduos com mais de 60 anos, residentes em Rio Claro, São Paulo, Brasil, dividida em G1 - sem acesso a tratamento odontológico conveniado $(n=55)$ e G2 com acesso a tratamento odontológico conveniado ( $n=$ 57). Os exames clínicos seguiram critérios da Organização Mundial da Saúde. A autopercepção foi avaliada usando o indice GOHAI (Geriatric Oral Health Assessment Index). O CPO-D foi de 29,13, maior no G1 ( $p=0,0091)$. A média de dentes presentes foi de 7,63, maior no G2 $(p=0,0131)$. As condições periodontais avaliadas apresentaram uma grande porcentagem de sextantes nulos (70,3\%), sendo que o CPI apontou o cálculo como a maior prevalência, e o PIP, as bolsas até $3 \mathrm{~mm}$. A porcentagem de indivíduos edêntulos foi de 45,5\%, maior no G1 ( $p=0,0142)$. Dentre os edêntulos, $69,6 \%$ usavam próteses totais superiores, e 42,9\%, inferiores. A média do GOHAI foi de 33,61, qualificando como positiva a percepção da saúde bucal e houve diferença apenas na dimensão física, com o maior valor no G2 ( $p=0,0154)$. A autopercepção da saúde bucal foi satisfatória, o que não pôde ser confirmado com os dados clínicos obtidos nos grupos.

Saúde Bucal; Auto-Imagem; Idoso

\section{Colaboradores}

D. D. Silva participou com a idéia original do tema, coleta dos dados e redação do texto. M. L. R. Sousa coordenou o processo de calibração (treinamento para a realização da pesquisa), contribuiu com a idéia original do tema e redação do texto. R. S. Wada analisou os resultados.

\section{Agradecimentos}

Ao Dr. Renato D. Lambertucci (Coordenador de Saúde Bucal), ao Centro Dia do Idoso, particularmente à Alice e Maria Augusta, aos coordenadores dos grupos de terceira idade (Melhor Idade) e à União dos Ferroviários Aposentados (UFA), em especial, ao Sr. Sérgio Harmitt e à Dra. Aline M. Joly, pela ajuda no agendamento dos voluntários, à presença fundamental dos voluntários, enfim, a todos que possibilitaram a realização desta pesquisa em Rio Claro.

\section{Referências}

1. Silva SRC, Castellanos-Fernandes RA. Autopercepção das condições de saúde bucal por idosos. Rev Saúde Pública 2001; 35:349-55.

2. World Health Organization. Oral health surveys: basic methods. $4^{\text {th }}$ Ed. Geneva: World Health Organization; 1997.

3. Mascarenhas AK. A comparison of oral health in elderly populations seeking and not seeking dental care. Spec Care Dentist 1999; 19:248-53.

4. Matthias RE, Atchison KA, Lubben JE, De Jong F, Scheweitzer SO. Factors affecting self-ratings of oral health. J Public Health Dent 1995; 55:197-204.

5. Locker D, Jokovic A. Three-year changes in selfperceived oral health status in an older Canadian population. J Dent Res 1997; 76:1292-7.

6. Locker D. Health outcomes of oral disorders. Int J Epidemiol 1995; 24 Suppl 1:S85-9.

7. Kressin NR, Atchison KA, Miller DR. Comparing the impact of oral disease in two populations of older adults: application of the Geriatric Oral Health Assessment Index. J Public Health Dent 1997; 57:224-32.

8. Reisine ST. The impact of dental conditions on social functioning and the quality of life. Ann Rev Public Health 1988; 9:1-19.

9. Atchison KA, Dolan TA. Development of the Geriatric Oral Health Assessment Index. J Dent Educ 1990; 54:680-7.

10. Atchison KA, Der-Martirosian C, Gift HC. Components of self-reported oral health and general health in racial and ethnic groups. J Public Health Dent 1998; 58:301-8.

11. Ministério da Saúde. Projeto SB 2003: condições de saúde bucal da população brasileira 2002-2003. 
Brasília: Departamento de Atenção Básica, Secretaria de Atenção à Saúde, Ministério da Saúde; 2004.

12. Paulander JA, Axelsson P, Lindhe J. Association between level of education and oral health status in 35-, 50-, 65 and 75-years-old. J Clin Periodontol 2003; 30:697-704.

13. Instituto Brasileiro de Geografia e Estatística. Censo demográfico 2000: primeiros resultados da amostra. http://www.ibge.gov.br (acessado em 23/Mai/2002).

14. Frias AC. Estudo de confiabilidade do levantamento epidemiológico de saúde bucal, Estado de São Paulo, 1998 [Dissertação de Mestrado]. São Paulo: Faculdade de Saúde Pública, Universidade de São Paulo; 2000.

15. Núcleo de Estudos e Pesquisas de Sistemas de Saúde, Secretaria de Estado da Saúde de São Paulo/Faculdade de Saúde Pública, Universidade de São Paulo. Levantamento das condições de saúde bucal - Estado de São Paulo, 1998: caderno de instruções. São Paulo: Faculdade de Saúde Pública, Universidade de São Paulo; 1998.

16. Schoueri Jr. R, Ramos LR, Papaléo-Netto M. Crescimento populacional: aspectos demográficos e sociais. In: Carvalho Filho ET, Papaléo-Netto M. Geriatria: fundamentos, clínica e terapêutica. São Paulo: Editora Atheneu; 1994. p. 9-29.

17. Paschoal SMP. Epidemiologia do envelhecimento. In: Papaléo-Netto M. Gerontologia. São Paulo: Editora Atheneu; 2002. p. 26-43.

18. Silva DD, Sousa MLR, Toledo R, Lisboa CM, Taglietta MF. Condições de saúde bucal em idosos na cidade de Piracicaba. Rev Assoc Paul Cir Dent 2002; 56:183-7.

19. Núcleo de Estudos e Pesquisas de Sistemas de Saúde, Secretaria de Estado da Saúde de São Paulo/Faculdade de Saúde Pública, Universidade de São Paulo. Levantamento epidemiológico em saúde bucal: Estado de São Paulo, 1998. São Paulo: Faculdade de Saúde Pública, Universidade de São Paulo; 1999.

20. Carneiro RMV. Saúde bucal em idosos institucionalizados na cidade de São Paulo: estudo epidemiológico e de autopercepção [Dissertação de Mestrado]. São Paulo: Faculdade de Saúde Pública, Universidade de São Paulo; 2001.

21. Angelillo IF, Sagliocco G, Hendricks SJH, Villari P. Tooth loss and dental caries in institutionalized elderly in Italy. Community Dent Oral Epidemiol 1990; 18:216-8.

22. Jokstad A, Ambjornsen E, Eide KE. Oral health in institutionalized elderly people in 1993 compared with in 1980. Acta Odontol Scand 1996; 54:303-8.

23. Padilha DMP, Souza MAL. Estado dentário e edentulismo observados em dois grupos de idosos do Brasil e da Inglaterra. Rev Odonto Cienc 1997; 12:67-85.

24. Castellanos-Fernandes RA, Silva SRC, Watanabe MGC, Pereira AC, Martildes MLR. Uso e necessidade de prótese dentária em idosos que demandam um centro de saúde. Rev Bras Odontol 1997; 54:107-10.

25. Silva SRC. Autopercepção das condições bucais em pessoas com 60 anos e mais de idade [Tese de Doutorado]. São Paulo: Faculdade de Saúde Pública, Universidade de São Paulo; 1999.
26. Cangussu MCT, Coelho EO, Castellanos-Fernandes RA. Condições de saúde bucal em adultos e idosos em Itatiba-SP, Brasil-2000. Rev Odontol UNESP 2001; 30:245-56.

27. Silva DD, Sousa MLR, Wada RS. Oral heath in adults and the elderly in Rio Claro, São Paulo, Brazil. Cad Saúde Pública 2004; 20:626-31.

28. Slade GD, Hoskin GW, Spencer AJ. Trends and fluctuations in the impact of oral conditions among older adults during a one year period. Community Dent Oral Epidemiol 1996; 24:317-21.

29. Hong-Ying W, Petersen PE, Jin-You B, Bo-Xue Z. The second national survey of oral health status of children and adults in China. Int Dent J 2002; 52:283-90.

30. Dolan TA. The sensitivity of the Geriatric Oral Health Assessment Index to Dental Care. J Dent Educ 1997; 61:36-46.

31. Suominen-Taipale AL, Nordblad A, Alanen P, Alha P, Koskinen S. Self-reported dental health, treatment need and attendance among older adults in two areas of Finland. Community Dent Health 2001; 18:20-6.

32. Rosa AGF, Castellanos-Fernandes RA, Pinto VG, Ramos LR. Condições de saúde bucal em pessoas de 60 anos ou mais no Município de São Paulo (Brasil). Rev Saúde Pública 1992; 26:155-60.

33. Núcleo de Estudos e Pesquisas de Sistemas de Saúde, Centro Técnico de Saúde Bucal, Secretaria de Estado da Saúde de São Paulo/ Faculdade de Saúde Pública, Universidade de São Paulo. Condições de saúde bucal no Estado de São Paulo em 2002. São Paulo: Faculdade de Saúde Pública, Universidade de São Paulo; 2002.

34. Shinkai RSA, Del Bel Cury AA. O papel da odontologia na equipe multidisciplinar: contribuindo para a atenção integral do idoso. Cad Saúde Pública 2000; 16:1099-109.

35. Steele JG, Sanders AE, Slade GD, Allen PF, Lahti S, Nuttall N, et al. How do age and tooth loss affect oral health impacts and quality of life? A study comparing two national samples. Community Dent Oral Epidemiol 2004; 32:107-14.

36. Locker D, Jokovic A, Clarke M. Assessing the responsiveness of measures of oral health-related quality of life. Community Dent Oral Epidemiol 2004; 32:10-8.

37. Jokovic A, Locker D. Dissatisfaction with oral health status in an older adult population. J Public Health Dent 1997; 57:40-7.

38. Atchison KA, Matthias RE, Dolan TA, Lubben JE, De Jong F, Scheweitzer SO, et al. Comparison of oral health ratings by dentists and dentate elders. J Public Health Dent 1993; 53:223-30.

39. Dolan TA, Peek CW, Stuck AE, Beck JC. Three-year changes in global oral health rating by elderly dentate adults. Community Dent Oral Epidemiol 1998; 26:62-9.

40. Silva DRAD. Percepção de condições de saúde bucal em adultos trabalhadores [Dissertação de Mestrado]. São Paulo: Faculdade de Saúde Pública, Universidade de São Paulo; 2000.

Recebido em 14/Jan/2004

Versão final reapresentada em 16/Dez/2004

Aprovado em 04/Mar/2005 